\title{
Upper-extremity function prospectively predicts adverse discharge and all-cause COPD readmissions: a pilot study
}

This article was published in the following Dove Press journal: International Journal of COPD

\author{
Hossein Ehsani ${ }^{1,2}$ \\ Martha Jane Mohler ${ }^{1-3}$ \\ Todd Golden² \\ Nima Toosizadeh ${ }^{1-3}$ \\ 'Department of Biomedical \\ Engineering, University of Arizona, \\ Tucson, AZ, USA; ${ }^{2}$ Arizona Center \\ on Aging, Department of Medicine, \\ University of Arizona, Tucson, AZ, \\ USA; ${ }^{3}$ Division of Geriatrics, General \\ Internal Medicine and Palliative \\ Medicine, Department of Medicine, \\ University of Arizona, Tucson, AZ, USA
}

Correspondence: Hossein Ehsani Department of Biomedical Engineering, University of Arizona, Bioscience Research Building, I60 I E Helen St, Tucson, AZ 857I9, USA

Tel +I 5206268780

Fax + I 52062658 ||

Email hehsani@aging.arizona.edu
Background: Frailty can inform management approaches for individuals with COPD. However, inpatient measures of frailty are seldom employed because they are time-consuming or inapplicable for bed-bound patients. We investigated the feasibility and potential of an innovative sensor-based upper-extremity function (UEF) test for frailty assessment in predicting adverse outcomes

Methods: Hospitalized patients with COPD-related exacerbations (aged $\geq 55$ years) were recruited and performed the UEF test within 24 hours of admission. UEF parameters were obtained and fed into our previously developed frailty model to calculate frailty status (non-frail, prefrail, and frail) and frailty score (0: extreme resilience to 1: extreme frailty). In-hospital (length of stay) and post-discharge (discharge disposition, 30-day exacerbation with treatment, and all-cause 30-day readmission) outcomes were collected. Associations between UEF frailty and outcomes were investigated using ANOVA and logistic models adjusted for demographic data. Results: In total, 42 patients were recruited. All participants were able to perform the UEF test. Based on UEF, participants were stratified into three groups of non-frail $(n=6$, frailty score $=0.18 \pm 0.09$ ), pre-frail $(n=14$, frailty score $=0.45 \pm 0.09)$, and frail $(n=22$, frailty score $=0.78 \pm 0.11$ ). Both frailty status and frailty score were significantly associated with unfavorable discharge disposition $(P<0.005)$ and all-cause 30-day readmission $(P<0.05)$. On the other hand, UEF frailty measures were associated with neither hospital length of stay $(P>0.5)$ nor 30-day exacerbation with treatment $(P>0.70)$. Age was only significantly associated with unfavorable discharge disposition $(P=0.048)$.

Conclusion: In agreement with previous work, the current findings underline the importance of measuring frailty for risk-stratification of COPD patients. The UEF was feasible and easily performed among all hospitalized COPD patients. In this study, we have shown that, using our quick and objective frailty measures, COPD patients can be prospectively risk-stratified in terms of unfavorable discharge disposition and all-cause 30-day readmissions.

Keywords: COPD, frailty, adverse health outcomes, early readmission, biomechanics, gait, wearable sensors

\section{Introduction}

Frailty is a state of increased risk of unfavorable outcomes when exposed to stressors, resulting in increased health care utilization, complications, institutionalization, disability, and death. COPD is one of the many chronic diseases associated with the frailty syndrome; both COPD and frailty share common risk factors and pathophysiology, including aging, sarcopenia, endocrine dysfunction, dysregulated inflammation, and smoking. ${ }^{1,2}$ The pooled prevalences of pre-frailty and frailty in COPD patients 
were reported in a 2018 meta-analysis as $56 \%$ and $19 \%$, respectively. ${ }^{3}$ Older adults with COPD have a two-fold increased risk of frailty, ${ }^{2,4,5}$ and severity of COPD (based on GOLD 2007 lung function ${ }^{6}$ ) is increased in those who are frail. ${ }^{2,4}$ The presence of frailty can change both the prognostic and management approaches of individuals with COPD. ${ }^{3}$

In the USA, hospitalizations for COPD-related exacerbations (COPDREs) account for more than 25\% (US $\$ 13.2$ billion) of the annual direct costs of COPD. ${ }^{7}$ The high 30-day readmission rate (one in five) among these patients significantly compounds the economic burden of COPD. ${ }^{8,9}$ Since up to $55 \%$ of these readmissions could be prevented, ${ }^{7}$ COPD was included as a condition under the Medicare Hospital Readmission Reduction Program (HRRP, 2014) to reduce all-cause readmission. ${ }^{10}$ Several factors have been identified as increasing the risk of all-cause early readmissions in patients admitted with COPDRE, one of which is the frailty syndrome. ${ }^{7}$ In general, frail older adults do not have sufficient physiological reserves, ${ }^{11}$ and therefore frail COPD patients may be more vulnerable to the negative impacts of COPD. Therefore, it has been hypothesized and verified that patients hospitalized with COPDRE who are prone to early readmission could be prospectively identified by measuring frailty. ${ }^{12-14}$

Single-item surrogate markers of motor function have been employed to measure frailty and, subsequently, to predict adverse health outcomes in patients admitted with COPDRE. ${ }^{14,15}$ The Six-Minute Walk Test distance (6MWD), as a measure of exercise capacity, has been successfully used to assess the severity of COPD, ${ }^{16,17}$ as well as the odds of mortality. ${ }^{15,18}$ However, because of its time and space requirements, ${ }^{19}$ this test may not be deemed practical in predicting adverse health outcomes in unwell bed-bound patients hospitalized with COPDRE. To obviate this limitation, in-hospital assessment of 4-m gait speed (4MGS), ${ }^{14}$ quadriceps (rectus femoris) crosssectional area as measured by ultrasound, and quadriceps maximal isometric strength have been studied as potential discriminators to risk-stratify patients hospitalized with COPDRE. ${ }^{20}$ These discriminators are relatively easy to measure and amenable to interventions; however, they still require walking ability, they are expensive (ultrasound), and none of them has been validated as a true predictor of frailty. Frailty is a multifaceted syndrome and different criteria should be incorporated into the frailty assessment procedure. ${ }^{1,21,22}$ For instance, when using the Fried phenotype for measuring physical frailty, five markers of weight loss, weakness, exhaustion, physical activity, and slowness have been considered. ${ }^{11}$ Focusing on a single surrogate marker for measuring physical frailty introduces significant error. ${ }^{7}$ For instance, while quadriceps size was introduced as a surrogate for general frailty and sarcopenia in previous work, ${ }^{20}$ quadriceps strength and the incremental and endurance shuttle walk tests were documented as inconsequential in the same study.

Inspired by the Fried phenotype, ${ }^{11}$ we have developed and validated a quick and objective screening tool for measuring frailty based on upper-extremity function (UEF). ${ }^{23,24}$ In this method, we use low-cost wearable motion sensors and mathematical modeling to quantify four criteria (slowness, exhaustion, weakness, and flexibility) in a 20 -second rapid arm flexion/extension test. In a cross-sectional study, we have previously shown the association of UEF parameters with 6MWD and pulmonary function measures (maximal inspiration and expiration pressures). ${ }^{25}$ Employing the Fried frailty phenotype as the gold standard, we have validated our UEF tool and developed two frailty indices (categorical, to identify non-frail, pre-frail, and frail, as well as a continuous score between 0 and 1 that is more sensitive to change over time). ${ }^{24}$ The physical assessment (including preparation/calibration) in the UEF test is easily performed in less than 1 minute, and post-processing to obtain the score is performed in less than 2 minutes. Considering these positive outcomes, the potential of the UEF test in predicting adverse health outcomes of bedbound patients with COPDRE merited further investigation.

The reported research was conducted in response to recommendations for longitudinal research examining temporal associations between COPD and frailty to further clarify the relationship between COPD and frailty. ${ }^{3}$ The objective of this study was to examine the feasibility of the UEF test to measure frailty category and frailty score among bed-bound patients hospitalized with COPDRE, as well as hospital-related outcomes (ie, hospital length of stay, in-hospital complications, unfavorable discharge disposition, 30-day readmission, and 30-day exacerbation requiring treatment). We hypothesized that the UEF test would be feasible among these patients, and that the UEF test could prospectively risk-stratify patients susceptible to poor outcomes.

\section{Patients and methods Definitions}

COPD was diagnosed in patients according to the GOLD criteria,${ }^{6}$ which are characterized by persistent airflow limitation, defined as a post-bronchodilator $\mathrm{FEV}_{1}$ equal to $70 \%$ of FVC. $^{6}$ 
Frailty syndrome categorical score (frail, pre-frail, nonfrail) and continuous score were established using UEF, which was validated using the Fried frailty phenotype. ${ }^{24}$

\section{Design}

We performed a 30-day longitudinal evaluation and descriptive feasibility study of patients admitted with COPDRE.

\section{Participants}

Hospitalized patients with COPD-related exacerbations were recruited from February 19, 2016 to May 6, 2016 from a large southwestern integrated academic health care system in the USA. Patients were diagnosed with COPDRE based on the hospital admission owing to worsening symptoms of COPD. The inclusion criteria were: 1) aged 55 years and older; 2) having consent capacity; and 3) capable of performing the repetitive fast elbow flexion. Exclusion criteria included 1) patients in intensive care; 2) ventilated patients; 3 ) diagnosed diseases associated with severe motor performance deficits, including stroke or Parkinson's disease/cogwheeling; and 4) notable bilateral upper-extremity musculoskeletal disorders (eg, bilateral fracture or rheumatoid arthritis with elbow or shoulder involvement). This study was approved by the University of Arizona Institutional Review Board.

\section{Procedures}

Prior to experiments, all participants were informed about the procedure and they signed the written consent form that was prepared based on the principles expressed in the Declaration of Helsinki. ${ }^{26}$ A summary of the procedures is shown in Figure 1.

\section{Clinical measures}

Baseline clinical measures, including the COPD Assessment Test (CAT; score range from 0 to 40: best to worst score) and Charlson Comorbidity Index (CCI; score range from 0 to 39 , representing the number of chronic conditions), were obtained from all participants. Based on chart reviews and telephone surveys, we determined the following health outcomes: discharge disposition (favorable: home; or unfavorable: home with care, rehabilitation center, skilled nursing facility, or death; see Joseph et al ${ }^{27,28}$ for more information regarding this classification), 30-day exacerbation requiring treatment, and 30-day readmission.

\section{UEF assessment}

Similar to our previously validated UEF method to measure frailty, ${ }^{23,24}$ wearable motion sensors (triaxial gyroscope sensors, sample frequency $=100 \mathrm{~Hz}$; BioSensics LLC,

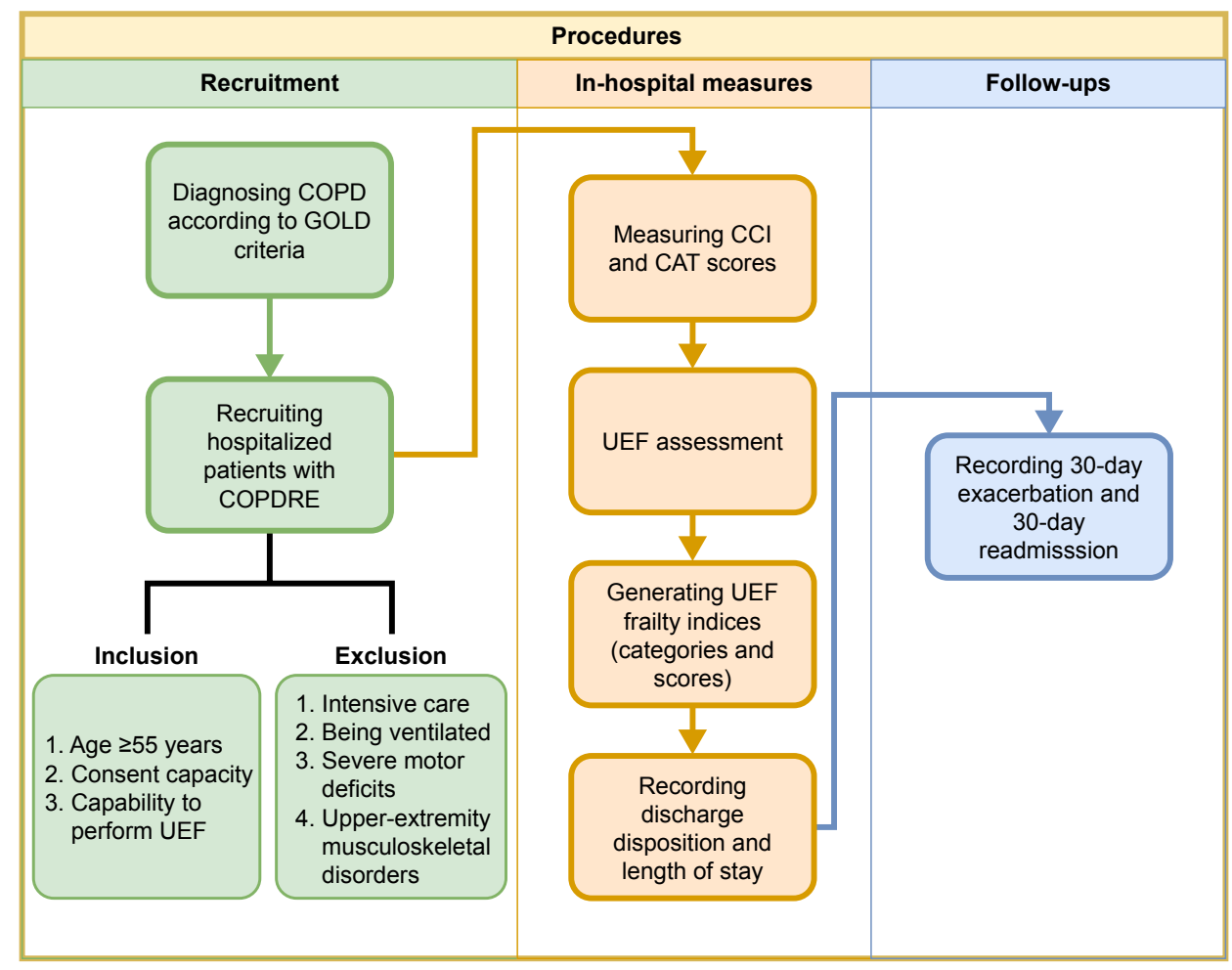

Figure I Summary of the procedures used in this study.

Abbreviations: CAT, COPD Assessment Test; CCI, Charlson Comorbidity Index; COPDRE, COPD-related exacerbations; UEF, upper-extremity function. 
Cambridge, MA, USA) were employed to capture forearm and upper-arm motion (Figure 2A). Each participant, while wearing the sensors in a seated position, performed a 20-second trial of rapid elbow flexion. Participants were instructed to repetitively and fully flex and extend their dominant elbow as quickly as possible. Although no physical limitation was imposed, participants were advised to keep their upper arm in a relatively fixed vertical position. Trained coordinators conducted all assessments, and to ensure consistency, all participants received the same instructions before measurement. After the protocol was explained to participants, they familiarized themselves with the protocol by performing a short practice run on their non-dominant arm prior to the actual experiment. Participants were encouraged only once before each test to perform the task as quickly as possible, and no further encouragements were given to them while performing the task.

Using sensor data (Figure 2B) and anthropometric information, the following UEF kinematic and kinetic measures were computed: 1) speed; 2) range of motion; 3) elbow moment; 4) speed reduction; 5) speed variability; and 6) flexion number (see Table 1 for definitions). Readers are referred to previous work ${ }^{23,24}$ for more details regarding the validation of UEF and parameter descriptions.

Using these quantities, we have previously developed two indices for measuring frailty. ${ }^{24}$ The first index, "Frailty status", is a categorical index, which presents the frailty status as non-frail, pre-frail, and frail. The second one, "frailty score", is a continuous score between 0 and 1 for measuring frailty, where " 0 " and " 1 " designate extreme resilience and extreme frailty, respectively (Figure 2C). In calculating the UEF score, each of the UEF parameters is assigned a weight based upon the parameter estimate values within an ordinal logistic model, with UEF parameters and demographic information as independent variables, and the Fried frailty categories as the dependent variable. ${ }^{24}$ The score has the following components (best score-worst score): ${ }^{24}$ slowness (0-0.48), exhaustion (0-0.22), weakness (0-0.08), flexibility

A

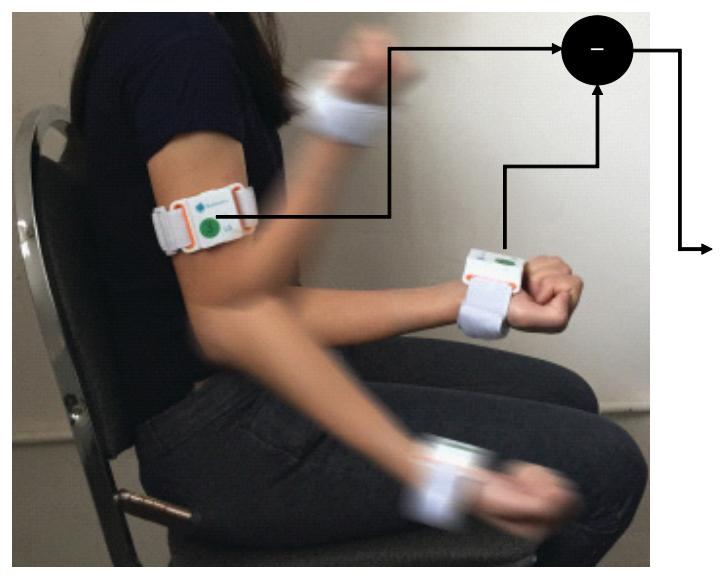

B
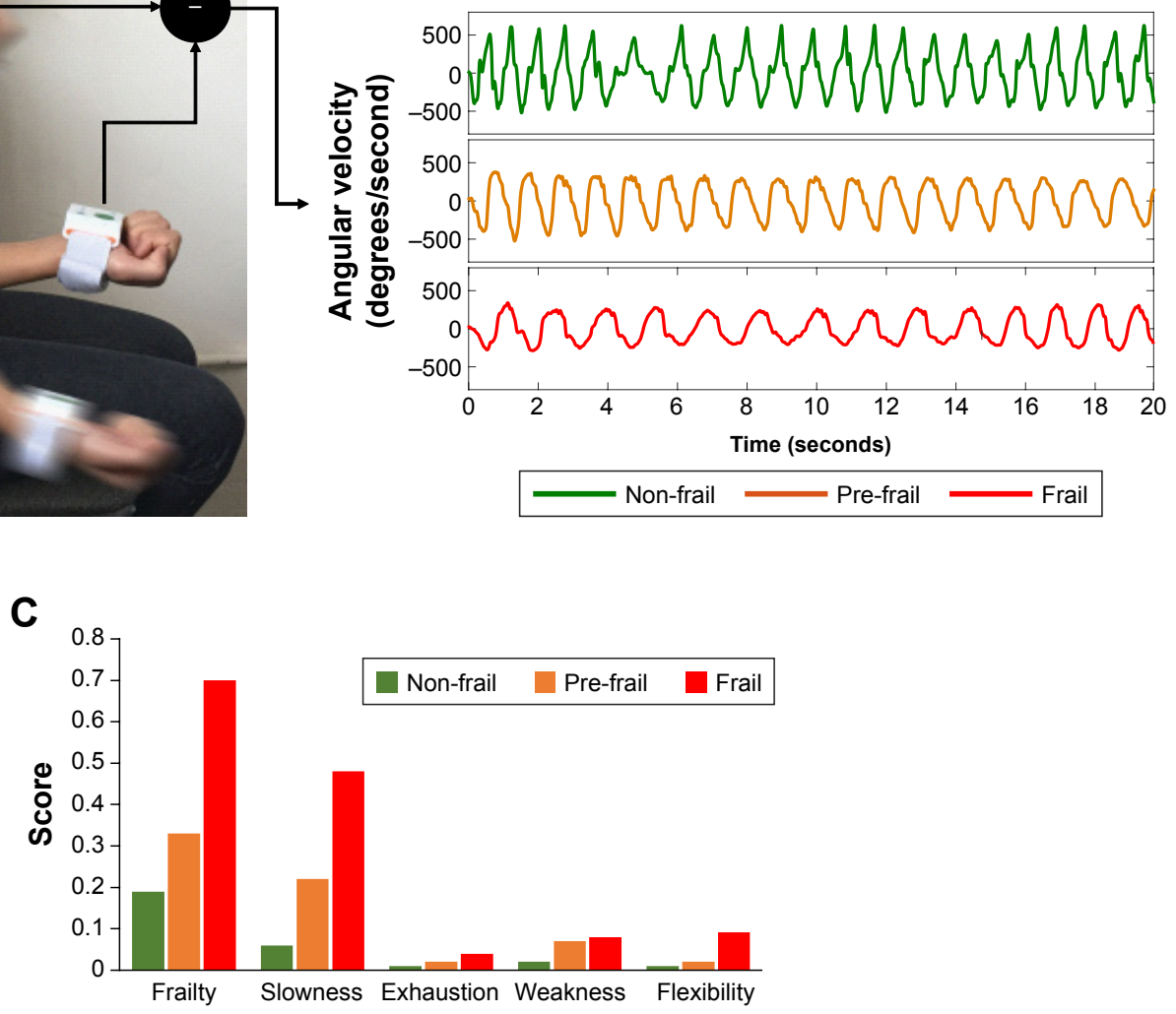

Figure 2 (A) UEF experimental set-up, (B) sensor outputs, and (C) UEF frailty score.

Notes: (A) Wearable motion sensors were used to capture forearm and upper-arm motion, and ultimately the elbow angular velocity during rapid elbow flexion/extension. Written consent was obtained from the participant to publish this figure. (B) Relative elbow angular velocity was obtained by subtracting sensor outputs. Results for one non-frail, one pre-frail, and one frail are presented. (C) The signals from section (B) were fed into our mathematical model to compute four criteria: slowness, exhaustion, weakness, and flexibility. The UEF frailty score was ultimately calculated based on these components.

Abbreviation: UEF, upper-extremity function. 
Table I Definitions of UEF parameters

\begin{tabular}{l|l|l}
\hline UEF parameter & Definition & Criterion \\
\hline Speed & Mean value of elbow angular velocity range (maximum minus minimum speed) \\
\hline Range of motion & Mean value of elbow flexion range & Slowness \\
\hline Moment & $\begin{array}{l}\text { Mean value of maximum moments on elbow within each flexion/extension estimated from moment } \\
\text { of inertia of forearm and hand, and elbow motion }\end{array}$ & Weakness \\
\hline Speed reduction & $\begin{array}{l}\text { Difference in angular velocity range between the last and the first } 5 \text { seconds of elbow flexion as a } \\
\text { percentage of the initial angular velocity range }\end{array}$ & Exhaustion \\
\hline Speed variability & Coefficient of variation of angular velocity range for 20 seconds & Exhaustion \\
\hline Flexion number & Number of flexion/extensions for 20 seconds & Slowness \\
\hline
\end{tabular}

Abbreviation: UEF, upper-extremity function.

$(0-0.20)$, and body mass index (BMI) (0-0.02). The UEF data obtained in this study were fed into these two models and both indices were obtained for all participants.

\section{Outcomes}

Outcomes included feasibility of performance of UEF (ie, ability to perform the test by those enrolled in the study), as well as hospital-related outcomes (ie, hospital length of stay, in-hospital complications, unfavorable discharge disposition, 30-day readmission [based on electronic health record review], and 30-day exacerbation requiring treatment [based on telephone follow-up]).

\section{Sample size}

Sample size estimation was based on previous UEF data (UEF score/parameter mean and SD among frailty groups), which determined that a sample size of at least 40 participants would be sufficient to achieve the required significance level of 0.05 in predicting 30-day readmission.

\section{Statistical analysis}

One-way ANOVA was used to assess between-group differences (ie, non-frail, pre-frail, and frail) among continuous sociodemographic parameters and adverse health outcomes (ie, frailty score, age, stature, weight, BMI, CAT, CCI, and mean hospital length of stay). Similarly, nominal logistic models were used to assess between-group differences among nominal variables (ie, sex, unfavorable discharge disposition, 30-day exacerbation with treatment, and 30-day readmission). In the case of health outcomes, we adjusted each model for age, sex, and BMI. Where appropriate, the corresponding Cohen's effect size was also determined. Further, separate models (ie, nominal logistic for nominal and ANOVA for continuous dependent variables) were developed to examine the association between frailty score (as the independent variables) with each of the health outcomes (as the dependent variable). All models were adjusted for age, sex, and BMI to account for possible influences of these parameters on health outcomes. In the case where UEF frailty score was a predictor of a health outcome, separate models were considered to examine the potency of each UEF component (ie, slowness, exhaustion, weakness, and flexibility) alone to predict the health outcomes. In each case, we also computed the area under the curve (AUC), using receiver operator characteristic (ROC) curves. The above analysis was repeated separately, considering each of the CAT and CCI scores as the independent variable. Moreover, to inspect the effect of age alone in predicting the adverse health outcomes, separate logistic models with age, sex, and BMI as the independent variables were considered. All analyses were conducted using JMP (version 11; SAS Institute, Cary, NC, USA), and statistical significance was indicated when $P \leq 0.05$.

\section{Results}

\section{Participants and adverse health outcomes}

In total, 42 patients were recruited for this study. All participants were able to perform the UEF assessment, and accordingly they were stratified into three groups of non-frail $(\mathrm{n}=6$, frailty score $=0.18 \pm 0.091$ ), pre-frail $(\mathrm{n}=14$, frailty score $=0.45 \pm 0.087)$, and frail $(\mathrm{n}=22$, frailty score $=0.78 \pm 0.11$ ). None of the sociodemographic information differed across groups $(P>0.29)$ (Table 2). Also, neither CAT nor CCI scores were significantly different among the groups $(P>0.70)$. Except for one participant in the pre-frail group, we documented the discharge disposition of all patients, and subsequently assessed the statistical significance of the unfavorable discharge disposition among the frailty groups $(P<0.01)$. There was one death within the 30-day follow-up period. We were unable to gather 30-day health data outcomes (30-day exacerbation and readmission) for two frail participants and one pre-frail participant. Among participants, 30-day readmission was 

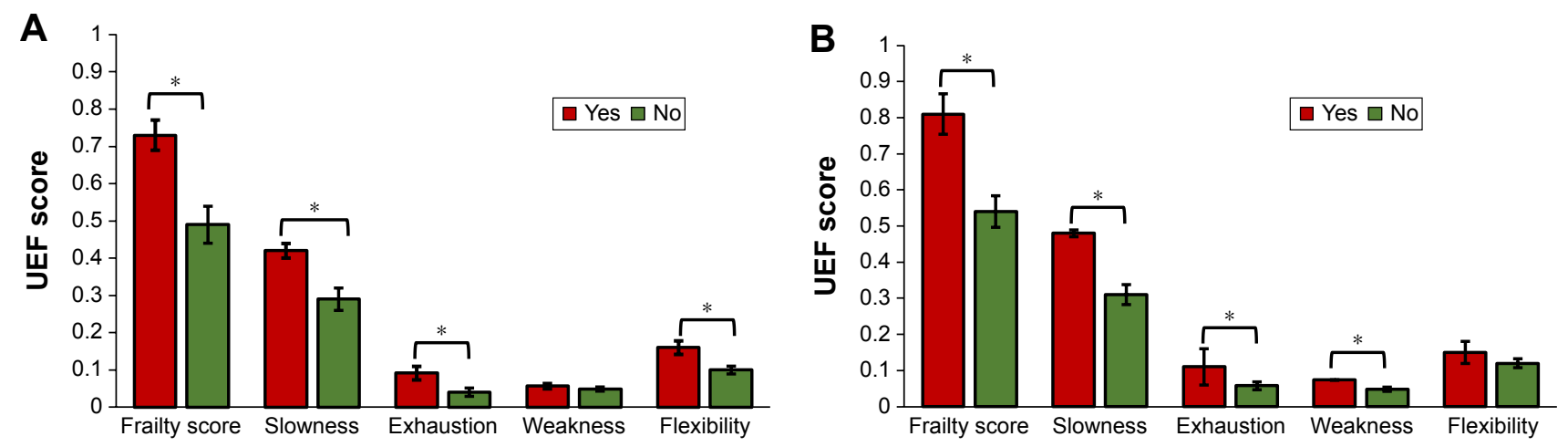

Figure 3 Potential of the frailty score and its components to risk-stratify COPD patients in terms of (A) unfavorable discharge disposition and (B) 30-day readmission. Notes: All models were adjusted for age, BMI, and sex. *Significant difference $(P \leq 0.05)$.

Abbreviations: BMI, body mass index; UEF, upper-extremity function.

significantly different among the frailty groups $(P=0.047)$, while 30-day exacerbation requiring treatment was nonsignificant $(P=0.77)$. The statistical details of sociodemographic and health outcome measures for frailty groups are shown in Table 2.

\section{Statistical models to predict adverse health outcomes}

As depicted in Table 3, when adjusted for age, sex, and BMI, the frailty score was a significant predictor of unfavorable discharge disposition $(P<0.01, \mathrm{AUC}=0.84)$ and 30-day readmission $(P=0.01, \mathrm{AUC}=0.84)$. On the other hand, similar to the frailty status (Table 2 ), neither 30-day exacerbation $(P=0.42)$ nor hospital length of stay $(P=0.44)$ was significantly associated with the frailty score. Among frailty score components, as shown in Figure 3, both slowness and exhaustion were predictors of unfavorable discharge disposition and 30-day readmission $(P<0.046$, mean AUC $=0.80)$. Although flexibility was a significant predictor of unfavorable discharge disposition $(P=0.041$, AUC $=0.75)$, it was nonsignificant for 30 -day readmission $(P=0.46)$. Conversely, while weakness was a predictor of 30-day readmission ( $P=0.039$, AUC $=0.80$ ), unfavorable discharge disposition was not associated with this component $(P=0.42)$. The ROC curves for these logistic models, predicting unfavorable discharge disposition and 30-day readmission, are shown in Figure 4.

When, instead of frailty score, the traditionally used CAT and $\mathrm{CCI}$ scores were considered, the models failed to predict any of the adverse health outcomes $(P>0.11)$. Further, age was significantly associated with only unfavorable discharge disposition $(P=0.048)$.

Table 2 Sociodemographic information and health outcome measures

\begin{tabular}{|c|c|c|c|c|c|}
\hline Variable & Non-frail group & Pre-frail group & Frail group & $P$-value & Effect size \\
\hline Number, $\mathrm{n}$ (\% of total) & $6(14)$ & $14(33)$ & $22(53)$ & - & - \\
\hline Frailty score, mean (SD) & $0.18(0.091)$ & $0.45(0.087)$ & $0.78(0.11)$ & $<0.0001 *$ & 2.21 \\
\hline Male, $n$ (\% of the group) & $3(50)$ & $8(54)$ & $17(78)$ & 0.29 & - \\
\hline Age (years), mean (SD) & $67(11)$ & $71(7)$ & $71(8)$ & 0.51 & 0.17 \\
\hline Stature $(\mathrm{cm})$, mean $(S D)$ & $171.82(12.80)$ & $169.42(9.68)$ & I74.80 (I0.03) & 0.32 & 0.24 \\
\hline Weight $(\mathrm{kg})$, mean (SD) & $75.01(20.56)$ & $78.48(19.91)$ & $75.59(22.03)$ & 0.91 & 0.07 \\
\hline BMI $\left(\mathrm{kg} / \mathrm{m}^{2}\right)$, mean $(\mathrm{SD})$ & $25.18(5.74)$ & $27.26(5.94)$ & $24.46(5.96)$ & 0.39 & 0.21 \\
\hline CAT (0-40 scale), mean (SD) & $24.17(7.02)$ & $22.7 \mathrm{I}(9.82)$ & $23.82(8.85)$ & 0.75 & 0.06 \\
\hline $\mathrm{CCl}$ (0-39 scale), mean (SD) & $3.67(1.37)$ & $3.14(1.46)$ & $3.14(1.67)$ & 0.70 & 0.12 \\
\hline Unfavorable discharge disposition, $\mathrm{n}$ (\% of the group) & $0(0)$ & $2(15)$ & $12(55)$ & $0.0038^{*}$ & - \\
\hline Hospital length of stay (days), mean (SD) & $6.67(4.92)$ & $5.77(3.32)$ & $7.09(3.90)$ & 0.56 & 0.15 \\
\hline 30-day exacerbation requiring treatment, $\mathrm{n}$ (\% of the group) & $2(33)$ & $4(31)$ & $8(40)$ & 0.77 & - \\
\hline 30-day readmission, $\mathrm{n}$ (\% of the group) & $0(0)$ & $0(0)$ & $4(20)$ & $0.047^{*}$ & - \\
\hline
\end{tabular}

Note: *Significant difference $(P \leq 0.05)$.

Abbreviations: BMI, body mass index; CAT, COPD Assessment Test; CCl, Charlson Comorbidity Index. 
Table 3 Association between the frailty score and adverse health outcomes

\begin{tabular}{|c|c|c|c|}
\hline Independent variables & $\begin{array}{l}\text { Dependent } \\
\text { variable }\end{array}$ & $\begin{array}{l}\chi^{2} \text { or } \\
\text { F-ratio }\end{array}$ & $P$-value \\
\hline $\begin{array}{l}\text { Frailty score }(0-1) \\
\text { Age (years) } \\
\text { Sex (male) } \\
\text { BMI }\left(\mathrm{kg} / \mathrm{m}^{2}\right)\end{array}$ & $\begin{array}{l}\text { Unfavorable } \\
\text { discharge } \\
\text { disposition }\end{array}$ & $\begin{array}{l}8.08 \\
2.93 \\
0.0257 \\
0.0045\end{array}$ & $\begin{array}{l}0.0045^{*} \\
0.087 \\
0.87 \\
0.95\end{array}$ \\
\hline $\begin{array}{l}\text { Frailty score }(0-\mathrm{l}) \\
\text { Age (years) } \\
\text { Sex (male) } \\
\text { BMI }\left(\mathrm{kg} / \mathrm{m}^{2}\right)\end{array}$ & $\begin{array}{l}\text { 30-day } \\
\text { exacerbation with } \\
\text { treatment }\end{array}$ & $\begin{array}{l}0.713 \\
1.12 \\
0.0583 \\
7.37 \mathrm{e}-6\end{array}$ & $\begin{array}{l}0.40 \\
0.29 \\
0.81 \\
0.99\end{array}$ \\
\hline $\begin{array}{l}\text { Frailty score }(0-\mathrm{I}) \\
\text { Age (years) } \\
\text { Sex (male) } \\
\text { BMI }\left(\mathrm{kg} / \mathrm{m}^{2}\right)\end{array}$ & $\begin{array}{l}\text { Hospital length of } \\
\text { stay }\end{array}$ & $\begin{array}{l}0.61 \\
0.00 \\
1.46 \\
0.54\end{array}$ & $\begin{array}{l}0.44 \\
1.00 \\
0.23 \\
0.47\end{array}$ \\
\hline $\begin{array}{l}\text { Frailty score }(0-\mathrm{I}) \\
\text { Age (years) } \\
\text { Sex (male) } \\
\text { BMI }\left(\mathrm{kg} / \mathrm{m}^{2}\right)\end{array}$ & 30-day readmission & $\begin{array}{l}6.47 \\
1.71 \\
0.0508 \\
1.22\end{array}$ & $\begin{array}{l}0.01 I^{*} \\
0.19 \\
0.82 \\
0.27\end{array}$ \\
\hline
\end{tabular}

Note: *Significant difference $(P \leq 0.05)$.

Abbreviation: BMI, body mass index.

\section{Discussion}

\section{Frailty and COPD}

Frailty is a prevalent syndrome among COPD patients and can inform both the prognostic and management approaches of individuals with COPD. In this study, using the UEF test, we objectively measured physical frailty among bed-bound hospitalized patients with COPDRE. We demonstrated that the UEF test was feasible to perform among bed-bound patients, and our frailty measures (status and score) were capable of risk-stratifying these patients in terms of unfavorable discharge disposition and all-cause 30-day readmission.

According to our UEF frailty status, more than half of the patients with COPD who participated in this study were identified as frail. This finding is in consonant with previous reports in which the prevalence of frailty among COPD patients was highlighted. ${ }^{2,4}$ It has been well documented that sarcopenia, age-associated muscle atrophy along with loss of skeletal muscle tissue quality and strength, is a critical component of the frailty syndrome. ${ }^{29-31}$ Low physical activity associated with shortness of breath in COPD patients leads to inactivity, ${ }^{32,33}$ and consequently a further decline in muscle strength ${ }^{34,35}$ and mobility problems. ${ }^{36}$ Therefore, it is not surprising that frailty syndrome is prevalent among COPD patients. It has been previously documented that in Medicare beneficiaries nearly $26 \%$ of early readmissions are caused by COPDRE. ${ }^{7,8}$ Therefore, considering the fact that frail elderly people are at higher risk of accelerated physical decline, ${ }^{37}$ one may argue that most all-cause adverse health outcomes seen in COPD patients can, in part, be explained by concomitant frailty syndrome. In the current study, we
A

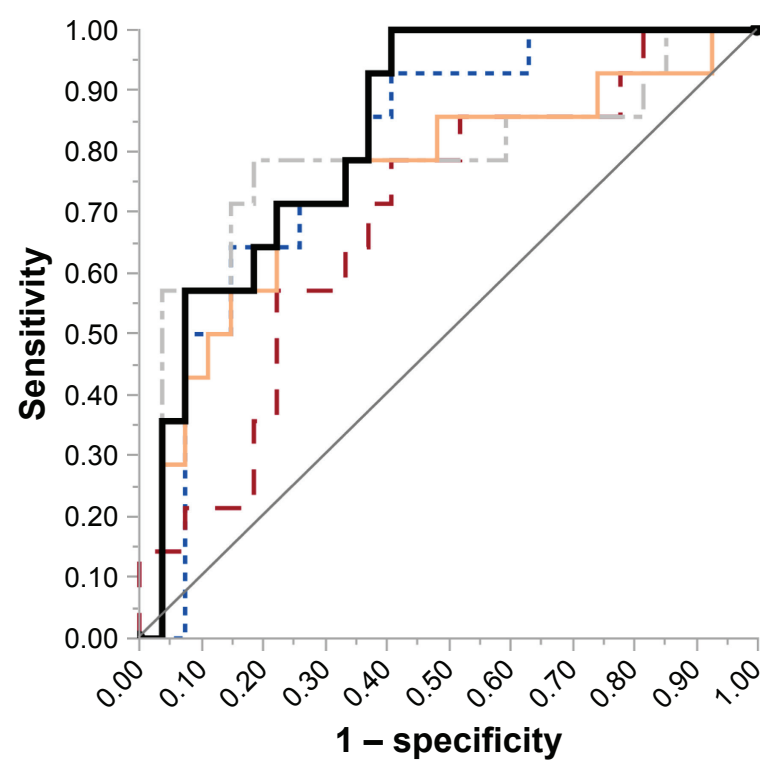

B

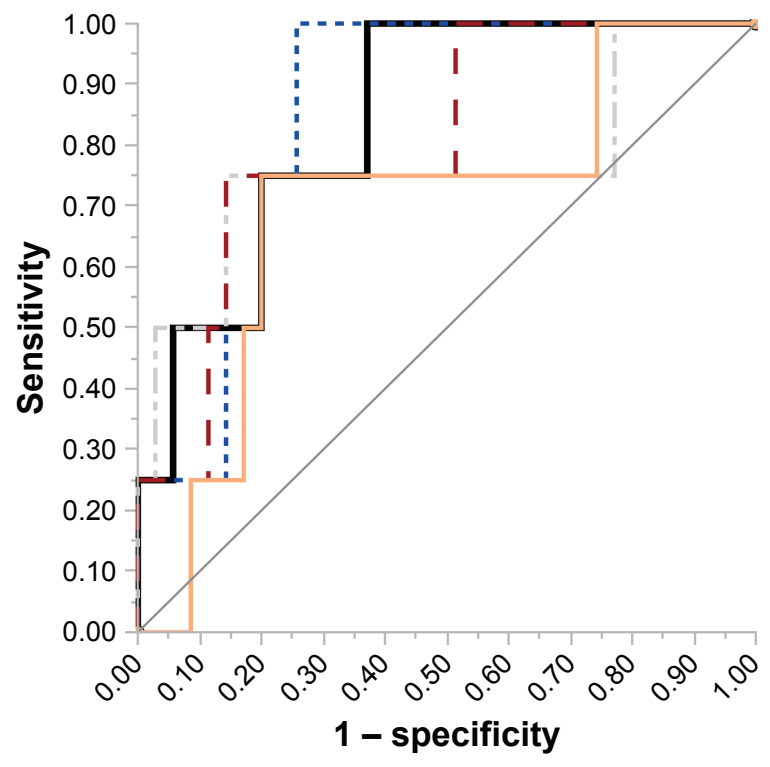

Frailty score - =- - Slowness $=-$ Exhaustion -- Weakness - Flexibility

Figure 4 ROC curves of the logistic models predicting (A) unfavorable discharge disposition and (B) 30-day readmission.

Note: All models were adjusted for age, BMI, and sex.

Abbreviations: BMI, body mass index; ROC, receiver operating characteristic. 
observed that all 30-day readmissions happened among the frail group (Table 2). Further, $86 \%$ of those who had unfavorable discharge disposition were also in the frail category, and the rest were pre-frail individuals (Table 2). These results accentuate the importance of frailty assessment to predict adverse health outcomes among COPD patients, especially those hospitalized with COPDRE, so as to better anticipate and meet the higher needs of this group.

Our categorical and continuous indices for measuring frailty were previously developed based on the Fried categorical index as the gold standard. ${ }^{24}$ Since our method was established based on a UEF, our indices could be obtained for unwell bed-bound patients hospitalized with COPDRE. Nonetheless, the same is not true for the Fried phenotype, as it requires gait assessment. ${ }^{11}$ Further, the increased risk of falls among frail individuals underlines the advantage of UEF over the Fried phenotype for measuring physical frailty. ${ }^{49,50}$ Another well-known method for assessing frailty is based on the Rockwood frailty index, in which frailty is defined as a state of increased vulnerability to adverse outcomes. ${ }^{22,38}$ Although the Rockwood index has been validated extensively for clinical use, it requires multiple pieces of clinical information and trained examiners to collect this information. Similar to Rockwood's method, Gobbens et al presented a conceptual integral model for frailty in which dynamic nature and multifactorial aspects of this syndrome were incorporated. ${ }^{21}$ In 2013, a modified version of this method was successfully employed to show the prevalence of frailty among COPD patients. ${ }^{4}$ In that cross-sectional study, a physical activity criterion was determined based on the data obtained from an ActiGraph attached to the right hip of participants for 7 days. ${ }^{4}$ Clearly, such an extensive measurement is not feasible for bed-bound patients within busy clinical settings. Although measuring frailty among patients hospitalized with COPDRE could be quite beneficial, in general, using a cumbersome and expensive method for this purpose would exacerbate health disparities. ${ }^{7}$ Our proposed objective method is quick and inexpensive, and it requires neither walking nor trained examiners. Although we have previously validated UEF using the Fried phenotype and the Rockwood frailty index, ${ }^{23,24,39}$ in the current study, for the first time, we longitudinally used this approach to assess adverse health outcomes among bed-bound patients hospitalized with COPDRE.

\section{Frailty factors among COPD patients}

As a remedy for the difficulties associated with multifactorial assessment of frailty in patients hospitalized with COPDRE, single-item surrogate markers of frailty were proposed. ${ }^{13,14,20}$ For instance, Kon et al showed that patients with smaller 4MGS on the day of discharge were more prone to all-cause 90 -day readmissions. ${ }^{14}$ Compared to the Fried phenotype, only the slowness criterion was considered in this surrogate marker, and the other criteria were neglected. Similarly, Greening et al demonstrated that quadriceps size as measured by ultrasound was independently associated with readmission within 1 year among patients hospitalized with COPDRE. ${ }^{20}$ While emphasizing sarcopenia as a critical component of frailty, the other factors of frailty, an intricate multifaceted syndrome, were dismissed in this single-item identifier. Our proposed frailty score comprised four criteria: slowness, exhaustion, weakness, and flexibility. As shown in Figures 3 and 4, it was possible to consider slowness alone to risk-stratify patients in terms of unfavorable discharge disposition and 30-day readmission; however, there existed other criteria that could also differentiate between patients (eg, exhaustion). On the other hand, as the frailty score performed better in terms of the AUC, it was suggested that the frailty score, reflecting the cumulative effect of its criteria, was a more potent tool to predict adverse health outcomes than one-item surrogate markers of frailty.

\section{Frailty and readmission prediction}

Risk-stratification of patients with COPDRE prone to early readmission at the time of index admission poses a serious challenge to hospitals. ${ }^{7}$ In the current study, employing frailty assessment with UEF in a small cohort, we showed that it was feasible to prospectively identify COPDRE patients prone to 30-day readmission. As in previous studies, ${ }^{14,20}$ the health status of COPD patients (measured by CAT score) was not associated with the all-cause early readmission $(P>0.4)$, suggesting that frailty might be a more dominant factor regarding this adverse health outcome than COPD severity. Similarly, in our population CCI was not a predictor of 30-day readmissions. These results, together with previous documented evidence regarding the importance of frailty in predicting adverse health outcomes, ${ }^{27,39-41}$ underlined the necessity of frailty assessments in hospitalized patients at the time of index admission.

\section{Frailty and discharge disposition prediction}

Discharge disposition is another key factor impacting the 30-day COPD readmission rate. It was previously demonstrated that patients discharged home without home care were more susceptible to all-cause early readmissions than those discharged to post-acute care (PAC), such as skilled nursing facilities and home care. ${ }^{8}$ Unfortunately, the 
decision-making process for the discharge destination might not happen in a timely manner, resulting in discharge delays and difficulties in coordination with PAC providers. ${ }^{42}$ In the current study, we demonstrated that UEF frailty indices were associated with an unfavorable discharge disposition. Of note, as age alone was also associated with the unfavorable discharge disposition $(P=0.048)$, logistic models corresponding to frailty prediction of discharge disposition were adjusted for age. Likewise, for 30-day readmission, neither the health condition of COPD patients $(P$-value for CAT score $=0.5$ ) nor the number of comorbidities $(P$-value for $\mathrm{CCI}=0.11$ ) was predictive of the discharge disposition. In addition to potential for early planning for the discharge destination, frailty assessment at the time of index admission could be beneficial in the triage assessment process and in the identification of COPD patients who may need more intensive therapy and enhanced attention during their index admission.

\section{Frailty, length of hospital stay, and exacerbation}

We did not find any association between hospital length of stay and frailty measures. In comparison to other conditions under the HRRP, such as congestive heart failure, there is little evidence-based guidance for hospitals to design efficient plans for patients hospitalized with COPDRE. ${ }^{7}$ As a result, it is possible that to reduce the risk of readmission, most admitted patients with COPDRE, regardless of their frailty condition, may be kept in hospitals for longer than necessary. This hypothesis should be further scrutinized in future studies by incorporating the diagnosis-related group of admission into the analysis. Of note, neither the health status of patients nor the number of comorbidities was predictive of the length of stay. Similarly, we could not predict 30-day exacerbation requiring treatment using our frailty measures. Although this item merits further investigations, it seems that, compared to frailty, the impact of other COPD-related factors (eg, the efficacy of prescribed antibiotics ${ }^{43}$ and the quality of nutrition care after discharge ${ }^{44}$ ) may be more influential.

\section{Limitations and future work}

This pilot study is limited by its small sample size. Results from the current study should be confirmed in larger samples, especially across multiple hospital settings. Further, the duration of follow-up was limited to 30 days, and, therefore, long-term evaluations of UEF for predicting adverse health outcomes is required. Moreover, in this study, additional COPD-related clinical details, eg, smoking index, were not included; however, they may be a useful line of inquiry in a future larger study.
Our UEF frailty measure does not incorporate measurement of cognitive decline, and, as with Fried, only focuses on physical frailty. Psychiatric conditions, including depression, cognitive impairment, and delirium, are key comorbidities in all-cause early readmissions in COPD patients, ${ }^{48}$ and the lack of inclusion of these factors is a limitation of this study.

UEF has been validated previously using the Fried frailty index, as well as the clinical Rockwood index..$^{40,41}$ In both studies, UEF was only validated for older adults, $\geq 65$ years old. In the current study, we showed the validity of UEF for predicting health outcomes for aging adults, aged $\geq 55$ years, with diagnosed COPD. In future studies, additional validation of UEF for aging adults hospitalized with other comorbidities is required to provide better understanding regarding functional performance and adverse health outcomes. Further, in future studies, computational models of the upper-extremity musculoskeletal system ${ }^{45-48}$ may be used to gain more insights into the weakness criterion and to improve the UEF frailty score among COPDRE patients.

\section{Conclusion}

In the current pilot study, using UEF, we objectively measured physical frailty among bed-bound patients hospitalized with COPDRE. All hospitalized patients were able to perform UEF tests during their admission. We showed that employing our frailty measures, ie, frailty status and frailty score, these patients could be prospectively risk-stratified in terms of unfavorable discharge disposition and all-cause 30-day readmissions. Neither patients' health status (as measured by CAT score) nor their number of comorbidities (as measured by CCI) was predictive of these adverse health outcomes. Although these findings provided evidence that UEF might be a better predictor of adverse post-discharge outcomes compared to CAT and CCI, these findings should be confirmed in a larger sample. The results of this study, together with previous work, underlined the importance of measuring physical frailty at the time of index admission among COPD patients. As a result, employing UEF as a quick and low-cost method for frailty assessment, in a frailty-prone population such as hospitalized COPD patients, would be greatly advantageous to both patients and health care systems.

\section{Author contributions}

HE contributed to the study concept and design, data collection, data analysis and interpretation of data, and preparation of the manuscript; JM contributed to the study concept and design, data analysis and interpretation of data, and preparation of the manuscript; TG contributed to data analysis and interpretation of data, acquisition of subjects, 
and preparation of the manuscript; and NT contributed to the study concept and design, data analysis and interpretation of data, and preparation of the manuscript. All authors contributed to data analysis, drafting and revising the article, gave final approval of the version to be published, and agree to be accountable for all aspects of the work.

\section{Disclosure}

The authors report no conflicts of interest in this work.

\section{References}

1. Angulo J, El Assar M, Rodríguez-Mañas L. Frailty and sarcopenia as the basis for the phenotypic manifestation of chronic diseases in older adults. Mol Aspects Med. 2016;50:1-32.

2. Lahousse L, Ziere G, Verlinden VJ, et al. Risk of frailty in elderly with COPD: a population-based study. J Gerontol A Biol Sci Med Sci. 2016; 71(5):689-695.

3. Marengoni A, Vetrano DL, Manes-Gravina E, Bernabei R, Onder G, Palmer K. The relationship between COPD and frailty: a systematic review and meta-analysis of observational studies. Chest. 2018; 154(1):21-40.

4. Park SK, Richardson CR, Holleman RG, Larson JL. Frailty in people with COPD, using the National Health and Nutrition Evaluation Survey dataset (2003-2006). Heart Lung. 2013;42(3):163-170.

5. Mittal N, Raj R, Islam EA, Nugent K. The frequency of frailty in ambulatory patients with chronic lung diseases. J Prim Care Community Health. 2016;7(1):10-15.

6. Rabe KF, Hurd S, Anzueto A, et al. Global strategy for the diagnosis, management, and prevention of chronic obstructive pulmonary disease: GOLD executive summary. Am J Respir Crit Care Med. 2007; 176(6):532-555.

7. Shah T, Press VG, Huisingh-Scheetz M, White SR. COPD readmissions: addressing COPD in the era of value-based health care. Chest. 2016; 150(4):916-926.

8. Shah T, Churpek MM, Coca Perraillon M, Konetzka RT. Understanding why patients with COPD get readmitted: a large national study to delineate the Medicare population for the readmissions penalty expansion. Chest. 2015;147(5):1219-1226.

9. Jencks SF, Williams MV, Coleman EA. Rehospitalizations among patients in the Medicare fee-for-service program. N Engl J Med. 2009; 360(14):1418-1428.

10. CMS.gov. Centers for Medicare \& Medicaid Services [homepage on the Internet. Hospital Readmissions Reduction Program (HRRP). Available from: http://www.cms.gov/Medicare/ Medicare-Fee-for-Service-Payment/AcuteInpatientPPS/ Readmissions-Reduction-Program.html. Accessed December 15, 2015.

11. Fried LP, Tangen CM, Walston J, et al. Frailty in older adults: evidence for a phenotype. J Gerontol A Biol Sci Med Sci. 2001;56(3): M146-M157.

12. Maddocks M, Kon SS, Canavan JL, et al. Physical frailty and pulmonary rehabilitation in COPD: a prospective cohort study. Thorax. 2016; 71(11):988-995.

13. Holland AE, Harrison SL, Brooks D. Multimorbidity, frailty and chronic obstructive pulmonary disease: are the challenges for pulmonary rehabilitation in the name? Chron Respir Dis. 2016;13(4): $372-382$.

14. Kon SS, Jones SE, Schofield SJ, et al. Gait speed and readmission following hospitalisation for acute exacerbations of COPD: a prospective study. Thorax. 2015;70(12):1131-1137.

15. Polkey MI, Spruit MA, Edwards LD, et al. Six-minute-walk test in chronic obstructive pulmonary disease: minimal clinically important difference for death or hospitalization. Am J Respir Crit Care Med. 2013; 187(4):382-386.
16. Casanova C, Cote CG, Marin JM, et al. The 6-min walking distance: long-term follow up in patients with COPD. Eur Respir J. 2007;29(3): $535-540$.

17. Cote CG, Pinto-Plata V, Kasprzyk K, Dordelly LJ, Celli BR. The 6-min walk distance, peak oxygen uptake, and mortality in COPD. Chest. 2007;132(6):1778-1785.

18. Pinto-Plata VM, Cote C, Cabral H, Taylor J, Celli BR. The 6-min walk distance: change over time and value as a predictor of survival in severe COPD. Eur Respir J. 2004;23(1):28-33.

19. ATS Committee on Proficiency Standards for Clinical Pulmonary Function Laboratories. ATS statement: guidelines for the six-minute walk test. Am J Respir Crit Care Med. 2002;166(1):111-117.

20. Greening NJ, Harvey-Dunstan TC, Chaplin EJ, et al. Bedside assessment of quadriceps muscle by ultrasound after admission for acute exacerbations of chronic respiratory disease. Am J Respir Crit Care Med. 2015;192(7):810-816.

21. Gobbens RJ, Luijkx KG, Wijnen-Sponselee MT, Schols JM. Towards an integral conceptual model of frailty. J Nutr Health Aging. 2010; 14(3):175-181.

22. Mitnitski AB, Mogilner AJ, Rockwood K. Accumulation of deficits as a proxy measure of aging. Scientific World Journal. 2001;1:323-336.

23. Toosizadeh N, Mohler J, Najafi B. Assessing upper extremity motion: an innovative method to identify frailty. J Am Geriatr Soc. 2015;63(6): 1181-1186.

24. Toosizadeh N, Wendel C, Hsu CH, Zamrini E, Mohler J. Frailty assessment in older adults using upper-extremity function: index development. BMC Geriatr. 2017;17(1):117.

25. Toosizadeh N, Berry C, Bime C, Najafi B, Kraft M, Mohler J. Assessing upper-extremity motion: an innovative method to quantify functional capacity in patients with chronic obstructive pulmonary disease. PLoS One. 2017;12(2):e0172766.

26. General Assembly of the World Medical Association. World Medical Association Declaration of Helsinki: ethical principles for medical research involving human subjects. $J$ Am Coll Dent. 2014; 81(3): 14 .

27. Joseph B, Toosizadeh N, Orouji Jokar T, Heusser MR, Mohler J, Najafi B. Upper-extremity function predicts adverse health outcomes among older adults hospitalized for ground-level falls. Gerontology. 2017;63(4):299-307.

28. Joseph B, Pandit V, Zangbar B, et al. Validating trauma-specific frailty index for geriatric trauma patients: a prospective analysis. $J$ Am Coll Surg. 2014;219(1):10-17.e1.

29. Marzetti E, Leeuwenburgh C. Skeletal muscle apoptosis, sarcopenia and frailty at old age. Exp Gerontol. 2006;41(12):1234-1238.

30. Bauer JM, Sieber CC. Sarcopenia and frailty: a clinician's controversial point of view. Exp Gerontol. 2008;43(7):674-678.

31. Cesari M, Landi F, Vellas B, Bernabei R, Marzetti E. Sarcopenia and physical frailty: two sides of the same coin. Front Aging Neurosci. 2014;6:192.

32. Garcia-Rio F, Lores V, Mediano O, et al. Daily physical activity in patients with chronic obstructive pulmonary disease is mainly associated with dynamic hyperinflation. Am J Respir Crit Care Med. 2009; 180(6):506-512.

33. Steele BG, Holt L, Belza B, Ferris S, Lakshminaryan S, Buchner DM. Quantitating physical activity in COPD using a triaxial accelerometer. Chest. 2000;117(5):1359-1367.

34. Roig M, Eng JJ, Macintyre DL, Road JD, Reid WD. Deficits in muscle strength, mass, quality, and mobility in people with chronic obstructive pulmonary disease. J Cardiopulm Rehabil Prev. 2011;31(2): 120-124.

35. Ferrucci L, Penninx BW, Volpato S, et al. Change in muscle strength explains accelerated decline of physical function in older women with high interleukin-6 serum levels. J Am Geriatr Soc. 2002;50(12): 1947-1954.

36. Butcher SJ, Meshke JM, Sheppard MS. Reductions in functional balance, coordination, and mobility measures among patients with stable chronic obstructive pulmonary disease. J Cardiopulm Rehabil. 2004;24(4):274-280. 
37. Fulop T, Larbi A, Witkowski JM, et al. Aging, frailty and age-related diseases. Biogerontology. 2010;11(5):547-563.

38. Searle SD, Mitnitski A, Gahbauer EA, Gill TM, Rockwood K. A standard procedure for creating a frailty index. BMC Geriatr. 2008; 8(1):24.

39. Toosizadeh N, Joseph B, Heusser MR, et al. Assessing upper-extremity motion: an innovative, objective method to identify frailty in older bed-bound trauma patients. J Am Coll Surg. 2016;223(2):240-248.

40. Guralnik JM, Simonsick EM, Ferrucci L, et al. A short physical performance battery assessing lower extremity function: association with self-reported disability and prediction of mortality and nursing home admission. J Gerontol. 1994;49(2):M85-M94.

41. McNallan SM, Singh M, Chamberlain AM, et al. Frailty and healthcare utilization among patients with heart failure in the community. JACC Heart Fail. 2013;1(2):135-141.

42. Lamantia MA, Scheunemann LP, Viera AJ, Busby-Whitehead J, Hanson LC. Interventions to improve transitional care between nursing homes and hospitals: a systematic review. J Am Geriatr Soc. 2010; 58(4):777-782.

43. Wang JX, Zhang SM, Li XH, Zhang Y, Xu ZY, Cao B. Acute exacerbations of chronic obstructive pulmonary disease with low serum procalcitonin values do not benefit from antibiotic treatment: a prospective randomized controlled trial. Int J Infect Dis. 2016;48:40-45.

44. van de Bool C, Rutten EPA, van Helvoort A, Franssen FME, Wouters EFM, Schols AMWJ. A randomized clinical trial investigating the efficacy of targeted nutrition as adjunct to exercise training in COPD. J Cachexia Sarcopenia Muscle. 2017;8(5):748-758.
45. Ehsani H, Rostami M, Gudarzi M. A general-purpose framework to simulate musculoskeletal system of human body: using a motion tracking approach. Comput Methods Biomech Biomed Engin. 2016;19(3): 306-319.

46. Ehsani H, Rostami M, Parnianpour M. A closed-form formula for the moment arm matrix of a general musculoskeletal model with considering joint constraint and motion rhythm. Multibody Syst Dyn. 2016; 36(4):377-403.

47. Moosavi F, Pasdar A, Ehsani H, Rostami M. An EMG-driven musculoskeletal model to predict muscle forces during performing a weight training exercise with a dumbbell. In: 19th Iranian Conference on Biomedical Engineering (ICBME), Tehran, Iran, 20-21 December 2012 Piscataway, NJ: IEEE.

48. Rafiee S, Ehsani H, Rostami M. A PCA-assisted EMG-driven model to predict upper extremities' joint torque in dynamic movements. In: 20th Iranian Conference on Biomedical Engineering (ICBME), Tehran, Iran, 18-20 December 2013. Piscataway, NJ: IEEE.

49. Toosizadeh N, Ehsani H, Miramonte M, Mohier J. Proprioceptive impairments in high fall risk older adults: the effect of mechanical calf vibration on postural balance. Biomed Eng Online. 2018;17(1):51.

50. Ehsani H, Mohler J, Marlinski V, Rashedi R, Toosizadeh N. The influence of mechanical vibration on local and central balance control. $J$ Biomech. 2018;71:59-66.
International Journal of COPD

\section{Publish your work in this journal}

The International Journal of COPD is an international, peer-reviewed journal of therapeutics and pharmacology focusing on concise rapid reporting of clinical studies and reviews in COPD. Special focus is given to the pathophysiological processes underlying the disease, intervention programs, patient focused education, and self management protocols.

\section{Dovepress}

This journal is indexed on PubMed Central, MedLine and CAS. The manuscript management system is completely online and includes a very quick and fair peer-review system, which is all easy to use. Visit http://www.dovepress.com/testimonials.php to read real quotes from published authors. 